Écrire

l'histoire

\section{Écrire l'histoire}

Histoire, Littérature, Esthétique

18 | 2018

Révolution

\title{
Allemagne nazie et révolution
}

Johann CНАРОUTOT, La Révolution culturelle nazie (2017)

\section{Marie-Bénédicte Vincent}

\section{OpenEdition}

Journals

Édition électronique

URL : http://journals.openedition.org/elh/1609

DOI : 10.4000/elh.1609

ISSN : 2492-7457

Éditeur

CNRS Éditions

\section{Édition imprimée}

Date de publication : 20 novembre 2018

Pagination : 201-204

ISBN : 978-2-271-12431-9

ISSN : 1967-7499

Référence électronique

Marie-Bénédicte Vincent, «Allemagne nazie et révolution », Écrire l'histoire [En ligne], 18 | 2018, mis en ligne le 20 novembre 2018, consulté le 16 février 2021. URL : http://journals.openedition.org/elh/1609 ; DOI : https://doi.org/10.4000/elh.1609

Ce document a été généré automatiquement le 16 février 2021.

Tous droits réservés 


\title{
Allemagne nazie et révolution
}

\author{
Johann Cнарочтот, La Révolution culturelle nazie (2017) \\ Marie-Bénédicte Vincent
}

\section{RÉFÉRENCE}

Johann СнаРочтот, La Révolution culturelle nazie, Paris, Gallimard (coll. « Bibliothèque des histoires »), 2017, $282 \mathrm{p}$.

1 Richard J. Evans écrivait déjà dans sa synthèse sur le régime nazi (Le Troisième Reich, 3 vol., Flammarion, 2009 pour la traduction française) que le nazisme avait été davantage une révolution culturelle qu'une révolution sociale. C'est dire que le titre choisi par Johann Chapoutot n'annonce pas en tant que tel un sujet neuf, mais s'inscrit au contraire dans les grandes questions qui traversent l'historiographie du nazisme. Son ouvrage rassemble plusieurs textes (déjà publiés dans diverses revues et réactualisés pour l'occasion) permettant de réfléchir à la manière dont les nazis refondent le système de valeurs (dans ses diverses dimensions : éthique, philosophique, historique, etc.), en Allemagne.

2 Sur le plan formel, le livre réunit onze études de cas classées en quatre parties proposant un parcours dans le système des représentations nazies pour en souligner la triste cohérence: premièrement, la dénonciation d'une dégénérescence de la race responsable d'une décadence séculaire allemande (aggravée depuis 1918); deuxièmement, la nécessité d'un retour à l'origine (celle de la pureté raciale); troisièmement, une refondation normative pour préparer le peuple à ses futurs combats; et, quatrièmement, le fonctionnement d'un système explicatif ayant sa propre logique tant dans les discours (par exemple celui d'Eichmann lors de son procès en 1961) que dans les œuvres culturelles (comme La Habanera, une comédie musicale de 1938 qui fut un succès cinématographique en Allemagne). Les sujets abordés sont extrêmement divers, allant de la philosophie (Quelle référence à Kant chez les nazis, puisque même Eichmann s'en réclame, en amont de son procès, quand il est interrogé par la police israélienne? Pourquoi Platon est-il érigé par les nazis en philosophe 
«allemand»?) au droit (Pourquoi le droit romain est-il remis en cause dès l'article 19 du programme du NSDAP de 1920 ?), à la sexualité "naturelle" (Comment les naissances illégitimes sont-elles justifiées? Pourquoi le mariage peut-il être dénoncé comme institution religieuse et bourgeoise quand il ne favorise pas la procréation? Jusqu'où va l'éloge de la monogamie chez les nazis?) ou à la terre (En quoi sa représentation est-elle profondément liée chez les nazis à l'idéologie raciale et au sang, et avec quelles conséquences terribles à partir de 1939 ?).

3 Sur le fond, l'ouvrage jette un pont entre les deux études précédentes de l'auteur, à savoir celle sur les usages et mésusages de l'Antiquité chez les nazis - qui voyaient l'Antiquité gréco-romaine comme l'acmé de la race nordique (Le Nazisme et l'Antiquité, PUF, 2012) - et celle sur la normativité nazie (La Loi du sang. Penser et agir en nazi, Gallimard, 2014). L'introduction présente du reste ce livre comme retraçant un parcours de recherches. La thèse de l'auteur peut être résumée comme suit: les nazis n'inventent rien; ils puisent dans divers éléments déjà développés avant eux depuis la fin $\mathrm{du} \mathrm{XIX}^{\mathrm{e}}$ siècle par les eugénistes, les auteurs racistes, les idéologues völkisch et pangermanistes (à l'instar du terme Lebensraum, dont on trouve la première occurrence en 1908 chez Dahl), les "anti-Lumières" (p. 75), mais aussi par certains médecins, pédagogues, philosophes ou juristes. Dès lors, l'originalité des nazis est de construire, à partir d'éléments épars, un système explicatif formellement cohérent, une "vision du monde» (Weltanschauung) qui réalise un renversement des valeurs "judéochrétiennes ", considérées comme responsables du déclin de la race nordique. Surtout, les nazis traduisent dans des législations et des politiques effectives ce nouveau système normatif, qui débouche sur les crimes de masse du régime et le génocide des juifs européens. La démarche historienne de Johann Chapoutot consiste à expliquer cette construction normative en partant des sources (des textes, des représentations, des images) et à en démontrer la redoutable efficacité quand elle est traduite en actes.

La discussion qui suit ne remet pas en cause les thèmes de cet ouvrage, dont la lecture s'avère toujours intéressante et le style très élégant. Elle veut plutôt pointer un problème du livre, qui est son manque de références aux travaux de recherche consacrés à ces sujets. L'appareil critique apparaît très peu développé : certaines études de cas se présentent sous la forme d'essais pratiquement sans notes en bas de page, tandis que celles qui adoptent explicitement la forme d'un article scientifique ne citent majoritairement que les sources consultées. Ce manque de référencement historiographique peut surprendre quand on connait la densité de certains champs de recherche, tels le cinéma allemand sous le nazisme, la politique familiale nazie ou le droit produit par les nazis et l'attitude des juristes allemands à partir de 1933. Faire du nazisme un objet d'histoire, ce qui est l'objectif revendiqué par l'auteur à la fin de son introduction (p. 19), nécessite bien sûr de rassembler et d'analyser un corpus original de sources - ce cahier des charges est du reste parfaitement rempli-, mais aussi de confronter ces sources et leurs interprétations aux résultats de la recherche en général, qui, sur un sujet aussi complexe que le nazisme, est forcément internationale et collective. La dimension historiographique, peut-être en raison du format choisi, fait ici défaut.

5 Ce qui suit relève donc d'une analyse personnelle visant à mettre en perspective historiographique l'important travail de Johann Chapoutot. Il nous semble en effet qu'il se tient à la croisée de deux courants de recherche. Le premier concerne la place centrale accordée depuis plusieurs années à l'idéologie pour expliquer à la fois son 
succès auprès des masses et la radicalité criminelle du régime. Il s'agit ici non pas de retracer toutes les étapes franchies depuis 1945, mais de rappeler que l'idéologie a d'abord été scrutée par les historiens «intentionnalistes » du nazisme, qui se sont penchés sur les plus hauts dirigeants et idéologues du régime (Hitler, Rosenberg, Goebbels notamment), avant d'être considérée comme moins centrale par les historiens dits "fonctionnalistes", qui privilégiaient l'analyse des structures du régime (organismes de pouvoir, bureaucraties, institutions) et celle des groupes sociaux embrigadés (ouvriers, fonctionnaires, jeunes, milieux professionnels, etc.). La discussion autour du terme de totalitarisme a aussi montré que la société n'était pas complètement idéologisée et que subsistaient, malgré la répression et les contraintes fortes imposées par la dictature, des niches d'opposition partielle ou radicale. On connaît la synthèse que propose Ian Kershaw au milieu des années 1990 entre les courants intentionnalistes et fonctionnalistes (termes devenus aujourd'hui obsolètes dans la recherche) avec son modèle de la domination charismatique (Hitler. Essai sur le charisme en politique, Gallimard, 1995 pour la traduction française), qui s'impose aux masses à la fois par sa dimension répressive et par l'adhésion qu'elle suscite. Dans cette veine, Peter Reichel explique l'attractivité du nazisme par l'esthétisation de la politique que le régime propose (La Fascination du nazisme, O.Jacob, 1993 pour la traduction française). Le nazisme représenterait ainsi pour certains une dictature du consentement. La Volksgemeinschaft, avec ses deux versants d'intégration et d'exclusion, revêtirait une réalité au-delà du slogan de mobilisation. Elle correspondrait à une société traversée par l'idéologie. Reste à comprendre comment la transformation de la société allemande en une telle Volksgemeinschaft a pu s'opérer. C'est pourquoi cette question reste aujourd'hui au centre des discussions scientifiques. Car d'autres chercheurs, tel Frank Bajohr, insistent moins sur l'idéologie que sur la corruption pour comprendre l'adhésion au régime de ceux qui espèrent en profiter matériellement d'une manière ou d'une autre.

6 Un autre champ s'est considérablement développé depuis les années 1990 : la recherche sur les Täter, "bourreaux » en français, perpetrators en anglais. La Täterforschung, qui s'est déployée en Allemagne comme à l'étranger notamment depuis les travaux de Christopher Browning (Des hommes ordinaires. Le 101 bataillon de réserve de la police allemande et la solution finale en Pologne, Les Belles Lettres, 1994 pour la traduction française), n'a pas seulement consisté à établir des profils de Täter (en montrant du reste que ceux-ci sont extrêmement divers sur le plan des générations, des origines géographiques et socio-économiques, des formations scolaires et professionnelles et des parcours de socialisation politiques). Ce courant a aussi révélé que l'idéologie n'était pas le seul facteur explicatif des comportements criminels: interviennent également la discipline, la solidarité corporative, le sadisme chez certains, l'indifférence chez d'autres, le conformisme chez la plupart, etc. On peut se reporter ici au très utile bilan historiographique sur les "acteurs de la barbarie » dressé par Gerhard Paul, qui a travaillé sur la Gestapo, dans Le Troisième Reich dans l'historiographie allemande. Lieux de pouvoirs, rivalités de pouvoirs, dirigé par Jean-Paul Cahn, Stefan Martens et Bernd Wegner, paru aux Presses universitaires du Septentrion en 2013 (p. 375 et suivantes). Néanmoins, on note que les derniers travaux publiés depuis, notamment par les chercheurs français tels Christian Ingrao (La Promesse de l'Est. Espérance nazie et génocide, Seuil, 2017) ou David Gallo (sur la SS), insistent particulièrement sur les effets criminels de l'idéologie incorporée par les Täter. En 
d'autres termes, l'idéologie, après avoir été minorée par les courants antérieurs, est à nouveau mise en exergue.

7 Pour revenir au livre de Johann Chapoutot, on peut donc le lire comme se situant au carrefour de ces deux directions de recherche, l'une s'intéressant au contenu de la "vision du monde » des nazis et à sa force d'attractivité dans la société, l'autre aux motivations des Täter entendus ici comme un groupe large d'acteurs impliqués dans l'élaboration et l'exécution des crimes du régime. Johann Chapoutot insiste, comme on l'a vu, sur la force de persuasion que représente la mise en cohérence de différents thèmes idéologiques choisis par les nazis parmi ceux qui étaient déjà présents dans la société. L'idéologie, devenue système, irrigue ensuite tous les domaines de la vie culturelle : philosophie, droit, vie artistique, etc. Il ne s'agit d'ailleurs plus d'une simple idéologie, mais d'un ensemble de valeurs beaucoup plus large. Reste à savoir comment ce système est accepté par une majorité. Ici, les travaux de psychologie sociale comme ceux d'Harald Welzer (Les Exécuteurs. Des hommes normaux aux meurtriers de masse, Gallimard, 2007 pour la traduction française) ont apporté des réponses.

8 Ainsi, ce sont, dans l'ouvrage de Johann Chapoutot, les contributions qui se situent à l'articulation entre la fabrication du système normatif nazi et sa réception dans la société qui nous ont paru les plus percutantes. Par exemple, l'analyse des hésitations, voire de la gêne des juristes nazis est particulièrement troublante, quand on voit que même un Hans Frank est déconcerté par la répudiation du droit romain par le NSDAP (p. 66). Une autre étude passionnante est celle, consacrée à la comédie musicale filmée de 1938 citée plus haut, qui montre comment, transposé dans un cadre exotique, le système normatif nazi peut être efficacement accepté par un public large alors qu'il s'agit du thème lourd de la contamination de la société par des agents pathogènes. Citons comme dernier exemple l'interprétation donnée par les nazis du traité de Versailles de 1919: il s'agit certes d'un sujet consensuel dans l'Allemagne de l'entredeux-guerres, mais le fait d'insérer le Diktat dans une tradition longue des traités de paix « français » qui seraient destinés à affaiblir l'Allemagne depuis celui de Westphalie en 1648 et d'inscrire cette généalogie dans les manuels scolaires permet de saisir comment un thème déjà présent dans la société trouve avec le nazisme une nouvelle acuité. En ce sens, il y a révolution culturelle quand une réception large de la Weltanschauung nazie a lieu.

\section{INDEX}

oeuvrecitee Révolution culturelle nazie (La) - (Johann Chapoutot, 2017) 


\section{AUTEURS}

\section{MARIE-BÉNÉDICTE VINCENT}

Marie-Bénédicte Vincent est maître de conférences habilitée à diriger des recherches en histoire contemporaine et spécialiste de l'histoire de l'Allemagne des $\mathrm{XIX}^{\mathrm{e}}$ et $\mathrm{XX}^{\mathrm{e}}$ siècles. Elle enseigne au département d'histoire de l'École normale supérieure ; elle est membre de l'Institut d'histoire moderne et contemporaine. Elle a notamment publié Histoire de la société allemande au $\mathrm{XX}^{e}$ siècle, vol. 1, Le premier XX ${ }^{e}$ siècle 1900-1949 (La Découverte, 2011) et Serviteurs de l'État. Les élites administratives en Prusse de 1871 à 1933 (Belin, 2006). Elle a dirigé deux ouvrages collectifs : La Dénazification (Perrin, coll. « Tempus », 2008) et Le Nazisme, régime criminel (Perrin, coll. « Tempus », 2015). La liste complète de ses publications peut être consultée sur <https:// histoire.ens.fr/-enseignants-du-departement-.html>. 